\title{
43. CALCIUM CARBONATE AND ORGANIC CARBON IN SAMPLES FROM DEEP SEA DRILLING PROJECT SITES 463, 464, 465, AND 466
}

\author{
Walter E. Dean, U.S. Geological Survey, Denver, Colorado
}

\section{METHODS}

Percent $\mathrm{CaCO}_{3}$ was determined in selected samples aboard the ship by the carbonate-bomb technique (Müller and Gastner, 1971). Results of these analyses are listed in Table 1 and plotted in Figures 1, 3,4 , and 5 as plus signs (+). Samples collected specifically for analyses of $\mathrm{CaCO}_{3}$ and organic carbon were analyzed at three shorebased laboratories. Concentrations of total carbon, organic carbon, and $\mathrm{CaCO}_{3}$ were determined in some samples at the DSDP sediment laboratory, using a Leco carbon analyzer, by personnel of the U.S. Geological Survey, under the supervision of T. L. Vallier (Table 2; plotted as square symbols in Figs. 1, 3, 4, and 5). Most of these samples were collected from lithologic units containing relatively high concentrations of organic carbon. Sample procedures are outlined in Boyce and Bode (1972). Precision and accuracy are both $\pm 0.3 \%$ absolute for total carbon, $\pm 0.06 \%$ absolute for organic carbon, and $\pm 3 \%$ absolute for $\mathrm{CaCO}_{3}$.

Concentrations of $\mathrm{CaCO}_{3}$ were determined in some samples with a Leco carbon analyzer at the University of Oslo under the supervision of Jörn Thiede (Table 3; plotted as circles in Figs. 1, 3, 4, and 5). The Oslo samples were mostly from lithologic units containing very low concentrations of organic carbon. The concentration of $\mathrm{CaCO}_{3}$ was calculated by multiplying the total carbon content by 8.33 . The accuracy of the method is $-2 \%$ to $+5 \%$ of the amount of $\mathrm{CaCO}_{3}$ present.

Concentrations of $\mathrm{CaCO}_{3}$ and organic carbon were determined at the University of Oslo on selected samples from Cores 69 through 72 of Hole 463 that were suspected to contain relatively high concentrations of organic carbon (Table 4; Fig. 2).

Concentrations of $\mathrm{CaCO}_{3}$ and organic carbon were determined at the U.S. Geological Survey, Denver, Colorado, in samples of midCretaceous limestone from Holes 463, 465A, and 466 that were suspected to contain relatively high concentrations of organic carbon (see Dean et al., this volume). Results of the USGS analyses are plotted as triangles in Figures 1, 2, 4, and 5. Concentrations of carbonate in samples analyzed by Müller et al. (this volume) are plotted in Figures 1, 3, 4, and 5 as X-symbols.

\section{RESULTS}

\section{Site $\mathbf{4 6 3}$}

The $\mathrm{CaCO}_{3}$ content of Lithologic Unit I $(0-450 \mathrm{~m}$ sub-bottom) is relatively constant at about $90 \%$, with a decrease to about $80 \%$ between 200 and 300 meters (Fig. 1). Contents of $\mathrm{CaCO}_{3}$ in Lithologic Unit II (450-585 m) range from 1 to $100 \%$, but most are greater than $50 \%$. Variations in concentration of $\mathrm{CaCO}_{3}$ in the multicolored limestone of Unit II is the result of variations in degree of silicification. A number of alternating couplets of light and dark limestones within this multicolored unit were specifically sampled to examine differences in content of $\mathrm{CaCO}_{3}$ between different-colored limestones. In general, the lower concentrations of $\mathrm{CaCO}_{3}$ are found

\footnotetext{
${ }^{1}$ Initial Reports of the Deep Sea Drilling Project, Volume 62
}

in the darker limestones (red, pink, and dark-green), and the higher concentrations are found in the lightercolored limestones (light-green). In Lithologic Unit III, $\mathrm{CaCO}_{3}$ is diluted by volcanic ash and organic matter, and this is reflected in the generally lower concentrations of $\mathrm{CaCO}_{3}$ in most samples analyzed from this unit. About half of the "limestone" samples analyzed from Lithologic Unit IV have concentrations of $\mathrm{CaCO}_{3}$ $<50 \%$. Examination of thin sections from this unit reveals a considerable amount of silicification, and concentrations of $\mathrm{SiO}_{2}$ range from 4 to $71 \%$, with an average of about $33 \%$ (Dean, this volume).

Most of the organic-carbon concentrations of samples from Lithologic Unit III (Cores 67-71) are high relative to concentrations commonly observed in pelagic carbonate sediments and rocks. Thin $(3-80 \mathrm{~cm})$ layers of dark, apparently organic-carbon-rich limestone occur within Unit III, especially in Cores 70 and 71 (Fig. 2). These layers contain as much as $7.5 \%$ organic carbon, but most samples from dark, organic-looking limestone layers contain less than $4.0 \%$ organic carbon. Dark colors are also due to the presence of dark tuffaceous material in the same unit. Therefore, interpretation of high organic content on the basis of dark color alone should be made with caution. Also, it is not known whether the association of organic matter with tuffaceous material in Lithologic Unit III is coincidental or if there is a causal relation between the two.

\section{Site $\mathbf{4 6 4}$}

The relatively high concentrations of $\mathrm{CaCO}_{3}$ (up to $20 \%$; Fig. 3) in the siliceous-fossil oozes of Lithologic Unit IA are due to minor amounts of nannofossils and recrystallized $\mathrm{CaCO}_{3}$, as observed in smear slides. As expected, the siliceous-fossil clays of Lithologic Unit IB and red and brown clays of Lithologic Unit II contain no $\mathrm{CaCO}_{3}$. Samples of silicified limestone associated with chert in Lithologic Unit III contain some $\mathrm{CaCO}_{3}$, indicating that the $\mathrm{CaCO}_{3}$ in these "limestones" probably has largely been replaced by $\mathrm{SiO}_{2}$.

\section{Site $\mathbf{4 6 5}$}

The $\mathrm{CaCO}_{3}$ content of Lithologic Unit I (nannofossil ooze; $0-276 \mathrm{~m}$ sub-bottom) is relatively constant between 88 and $96 \%$ (Fig. 4). The concentrations of $\mathrm{CaCO}_{3}$ in Lithologic Unit II (laminated limestone) are generally lower and more variable, because of varying degrees of silicification within this unit $\left(\mathrm{SiO}_{2}\right.$ varies between 1 and $25 \%$; Dean, this volume). Concentrations 
Table 1. Results of shipboard carbonate-bomb analyses, Sites 463, 464,465 , and 466 .

\begin{tabular}{|c|c|c|c|}
\hline $\begin{array}{c}\text { Sample } \\
\text { (interval in } \mathrm{cm} \text { ) }\end{array}$ & $\begin{array}{c}\text { Depth } \\
\text { Sub-bottom } \\
\text { (m) }\end{array}$ & $\begin{array}{c}\mathrm{CaCO}_{3} \\
\text { (bomb) } \\
(\%)\end{array}$ & Lithology \\
\hline $463-1-3,70-71$ & 3.7 & $>90$ & Nannofossil ooze \\
\hline $2-3,85-86$ & 9.4 & 89 & Nannofossil ooze \\
\hline $3-3,89-90$ & 18.9 & 79 & Foraminifer ooze \\
\hline $4-3,73-74$ & 28.2 & 88 & Foraminifer-nannofossil ooze \\
\hline $5-1,83-84$ & 34.8 & 89 & Nannofossil ooze \\
\hline $6-3,70-71$ & 41.7 & 96 & Foraminifer-nannofossil ooze \\
\hline $7-3,100-101$ & 47.5 & 91 & Nannofossil chalk \\
\hline $8-3,60-61$ & 56.6 & 91 & Foraminifer-nannofossil chalk \\
\hline $9-3,33-34$ & 65.8 & 91 & Foraminifer-nannofossil chalk \\
\hline $10-3,81-82$ & 75.8 & 69 & Foraminifer-nannofossil chalk \\
\hline $11-3,31-32$ & 84.8 & 91 & Foraminifer-nannofossil chalk \\
\hline $12-6,85-86$ & 99.4 & 91 & Foraminifer-nannofossil chalk \\
\hline $13-3,55-56$ & 104.1 & 91 & Foraminifer-nannofossil chalk \\
\hline $14-2,100-101$ & 112.5 & 91 & Foraminifer-nannofossil chalk \\
\hline $15-3,80-81$ & 123.3 & 91 & Foraminifer-nannofossil chalk \\
\hline $16-4,20-21$ & 133.7 & 89 & Foraminifer-nannofossil chalk \\
\hline $17-3,100-101$ & 142.5 & 91 & Foraminifer-nannofossil chalk \\
\hline $19-3,127-128$ & 161.8 & 92 & Foraminifer-nannofossil chalk \\
\hline $20-1,57-59$ & 167.6 & 91 & Foraminifer-nannofossil chalk \\
\hline $21-3,104-105$ & 180.5 & 100 & Foraminifer-nannofossil chalk \\
\hline $22-3,104-105$ & 190.0 & 89 & Foraminifer-nannofossil chalk \\
\hline $23-1,131-132$ & 196.8 & 96 & Nannofossil-foraminifer chalk \\
\hline $24-3,34-35$ & 202.8 & 88 & Foraminifer-nannofossil chalk \\
\hline $25-2,28-29$ & 206.8 & 86 & Foraminifer-nannofossil chalk \\
\hline $26-3,20-21$ & 217.2 & 86 & Foraminifer-nannofossil chalk \\
\hline $27-2,35-36$ & 225.9 & 79 & Foraminifer-nannofossil chalk \\
\hline $29-1,58-59$ & 243.6 & 81 & Nannofossil-foraminifer chalk \\
\hline $30-2,35-36$ & 254.4 & 76 & Nannofossil chalk \\
\hline $31-1,12-13$ & 262.1 & 81 & Nannofossil limestone \\
\hline $33-2,107-108$ & 283.6 & 88 & Nannofossil chalk \\
\hline $34-3,20-21$ & 293.7 & 86 & Nannofossil foraminifer chalk \\
\hline $48-3,70-71$ & 427.2 & 91 & Nannofossil chalk \\
\hline $55-1,64-65$ & 481.1 & 87 & Limestone \\
\hline $56-1,93-94$ & 490.9 & 88 & Nannofossil limestone \\
\hline $57-1,44-45$ & 499.9 & 84 & Nannofossil limestone (light-green) \\
\hline $58-2,10-11$ & 510.6 & 70 & Nannofossil limestone (pink) \\
\hline $59-1,10-11$ & 518.6 & 80 & Nannofossil limestone (dark-green) \\
\hline $59-2,16-17$ & 520.2 & 66 & Nannofossil limestone (dark-green) \\
\hline $60-2,42-44$ & 529.9 & 88 & Nannofossil limestone (pink) \\
\hline $62-1,91-92$ & 538.4 & 57 & Nannofossil limestone (dark-green) \\
\hline $63-1,47-48$ & 547.5 & 79 & Nannofossil limestone (light-green) \\
\hline $63-1,52-53$ & 547.5 & 62 & Nannofossil limestone (red) \\
\hline $64-2,34-35$ & 558.3 & 85 & Nannofossil limestone (light-green) \\
\hline $64-2,52-53$ & 553.5 & 69 & Nannofossil limestone (pink) \\
\hline $65-2,16-17$ & 567.7 & 85 & Nannofossil limestone (light-green) \\
\hline $65-2,27-28$ & 567.8 & 73 & Nannofossil limestone (pink) \\
\hline $66-3,64-65$ & 579.1 & 77 & Nannofossil limestone (light-gray) \\
\hline $66-3,79-80$ & 579.3 & 68 & Nannofossil limestone (light-green) \\
\hline $67-1,24-25$ & 585.2 & 58 & Nannofossil limestone (light-gray, cherty) \\
\hline $69-2,18-19$ & 605.7 & 85 & Nannofossil limestone (bluish-white) \\
\hline $69-2,30-31$ & 605.8 & 42 & Volcanic ash (greenish-black) \\
\hline $71-3,21-22$ & 626.2 & 36 & Limestone (light-green) \\
\hline $73-4,75-76$ & 647.3 & 8 & Limestone (bluish-gray) \\
\hline $73-4,90-91$ & 647.4 & 30 & Limestone (dark-gray) \\
\hline $74-2,13-14$ & 653.1 & 86 & Limestone (light-gray) \\
\hline $76-1,46-49$ & 670.9 & 41 & Limestone (light-gray) \\
\hline $77-1,110-111$ & 681.1 & 22 & Limestone (white) \\
\hline $79-1,34-35$ & 699.3 & 4 & Limestone (white; siliceous) \\
\hline $81-2,8-9$ & 712.1 & 90 & Limestone (light-gray, sandy) \\
\hline $82-1,50-51$ & 718.5 & 90 & Limestone (light-gray) \\
\hline $83-1,77-78$ & 728.3 & 47 & Limestone (white) \\
\hline $84-1,134-135$ & 738.3 & 75 & Limestone (white) \\
\hline $85-1,99-100$ & 747.5 & 87 & Limestone (light-gray, clastic) \\
\hline $464-2-3,135-136$ & 7.85 & 11.5 & Diatom-radiolarian ooze \\
\hline $3-3,89-90$ & 14.90 & 11.5 & Clayey radiolarian ooze \\
\hline $4-3,80-81$ & 26.30 & 0.0 & Siliceous clay \\
\hline $5-3,85-86$ & 35.85 & 0.0 & Siliceous clay \\
\hline $6-5,65-66$ & 48.15 & 0.0 & Brown clay \\
\hline $7-2,145-146$ & 53.95 & 0.0 & Brown clay \\
\hline $9-6,74-75$ & 78.25 & 0.5 & Brown clay \\
\hline $10-2,136-137$ & 82.36 & 1.5 & Red clay \\
\hline $11-1,11-12$ & 89.11 & 0.0 & Porcellanite \\
\hline $12-1,22-23$ & 98.72 & 19 & Siliceous limestone \\
\hline $13, \mathrm{CC}, 9-10$ & 117.5 & 26.5 & Siliceous limestone \\
\hline $465-2-2,33-34$ & 2.83 & 81 & Nannofossil ooze \\
\hline $2-5,33-34$ & 7.33 & 90 & Nannofossil ooze \\
\hline $3-3,66-67$ & 14.16 & 90 & Nannofossil ooze \\
\hline $4-3,59-60$ & 23.60 & 88 & Nannofossil ooze \\
\hline $5-3,109-110$ & 33.59 & 88 & Nannofossil ooze \\
\hline $6-3,99-100$ & 43.00 & 94 & Nannofossil ooze \\
\hline $10-5,16-17$ & 83.16 & 90 & Nannofossil ooze \\
\hline $465 \mathrm{~A}-1-1,55-56$ & 39.55 & 93 & Nannofossil ooze \\
\hline $3-2,70-71$ & 60.20 & 90 & Nannofossil ooze \\
\hline $3-4,70-71$ & 63.20 & 89 & Nannofossil ooze \\
\hline $9-4,82-84$ & 120.32 & 81 & Foraminifer-nannofossil ooze \\
\hline $10-3,64-65$ & 128.14 & 96 & Foraminifer-nannofossil ooze \\
\hline
\end{tabular}

Table 1. (Continued).

\begin{tabular}{|c|c|c|c|}
\hline $\begin{array}{c}\text { Sample } \\
\text { (interval in } \mathrm{cm} \text { ) }\end{array}$ & $\begin{array}{l}\text { Depth } \\
\text { Sub-bottom } \\
\text { (m) }\end{array}$ & $\begin{array}{c}\mathrm{CaCO}_{3} \\
\text { (bomb) } \\
(\%)\end{array}$ & Lithology \\
\hline $11-3,60-62$ & 137.60 & 96 & Nannofossil ooze \\
\hline $12-2,70-72$ & 145.70 & 94 & Nannofossil ooze \\
\hline $15-3,20-21$ & 175.20 & 93 & Foraminifer-nannofossil ooze \\
\hline $16-4,37-38$ & 186.37 & 96 & Foraminifer-nannofossil ooze \\
\hline $17-1,20-22$ & 191.20 & 93 & Foraminifer-nannofossil ooze \\
\hline $18-2,4-5$ & 202.04 & 88 & Foraminifer-nannofossil ooze \\
\hline $19-2,92-93$ & 212.42 & 94 & Nannofossil ooze \\
\hline $20-1,110-111$ & 220.60 & 94 & Foraminifer-nannofossil ooze \\
\hline $21-4,75-76$ & 234.25 & 92 & Foraminifer-nannofossil chalk \\
\hline $26-1,44-45$ & 276.94 & 93 & $\begin{array}{l}\text { Laminated limestone } \\
\text { (bottom of turbidite) }\end{array}$ \\
\hline $26-1,49-50$ & 276.99 & 94 & $\begin{array}{l}\text { Laminated limestone } \\
\text { (top of a graded turbidite) }\end{array}$ \\
\hline $27-2,89-90$ & 288.39 & 92 & Laminated limestone \\
\hline $27-2,110-111$ & 288.60 & 20 & Chert \\
\hline $28-1,111-112$ & 296.61 & 93 & Laminated limestone \\
\hline $28-1,123-124$ & 296.73 & 70 & Black limestone band \\
\hline $29-1,93-94$ & 305.93 & 58 & Gray, massive limestone \\
\hline $466-1-1,20-21$ & 0.20 & 79 & Foraminifer-nannofossil ooze \\
\hline $1-1,62-63$ & 0.62 & 74 & Radiolarian-nannofossil ooze \\
\hline $1-3,120-120$ & 4.20 & 82 & Nannofossil ooze (light-gray) \\
\hline $2-2,22-23$ & 9.72 & 87 & Nannofossil ooze (light-gray) \\
\hline $2-6,22-23$ & 15.72 & 70 & Nannofossil ooze (green-gray) \\
\hline $3-1,100-101$ & 18.50 & 78 & Nannofossil ooze (green-gray) \\
\hline $3-1,120-121$ & 18.70 & 89 & Nannofossil ooze (white) \\
\hline $4-2,34-35$ & 28.84 & 89.5 & Nannofossil ooze (white) \\
\hline $5-2,42-43$ & 38.42 & 89.5 & Nannofossil ooze (white) \\
\hline $5-5,42-43$ & 42.92 & 92 & Nannofossil ooze (white) \\
\hline $6-3,85-86$ & 49.85 & 90 & Nannofossil ooze (light-gray) \\
\hline $6-6,85-86$ & 54.36 & 66 & Nannofossil ooze (white) \\
\hline $7-2,10-11$ & 57.10 & 90 & Nannofossil ooze (white) \\
\hline $7-6,10-11$ & 63.10 & 90 & Nannofossil ooze (light-gray-brown) \\
\hline $8-3,90-91$ & 68.90 & 93 & Nannofossil ooze (pale-brown) \\
\hline $8-6,90-91$ & 73.40 & 82 & Nannofossil ooze (dark yellow-brown) \\
\hline $9-3,60-61$ & 78.10 & 91 & Nannofossil ooze (pale brown) \\
\hline $11-1,52-54$ & 88.52 & 98 & Nannofossil ooze (white) \\
\hline $12-1,29-30$ & 93.79 & 97 & Nannofossil ooze (white) \\
\hline $13-2,100-101$ & 105.50 & 97 & Nannofossil ooze (white) \\
\hline $14-1,74-75$ & 113.24 & 93 & Nannofossil ooze (white) \\
\hline $15-3,47-48$ & 125.47 & 93 & Nannofossil ooze (white) \\
\hline $16-2,109-110$ & 134.09 & 93 & Nannofossil ooze (white) \\
\hline $17-1,77-78$ & 141.77 & 96 & Nannofossil ooze (white) \\
\hline $29-1,60-61$ & 255.60 & 73 & Limestone (dark olive-gray) \\
\hline $30-1,10-11$ & 264.60 & 93 & Limestone (olive-gray) \\
\hline $30-1,59-60$ & 265.19 & 80 & Limestone (olive-gray) \\
\hline $30-1,71-72$ & 315.21 & 90 & Laminated limestone \\
\hline $31-1,15-16$ & 324.15 & 93 & Laminated limestone \\
\hline $32-1,94-95$ & 334.44 & 81 & Laminated limestone \\
\hline $33-1,65-66$ & 343.65 & 88 & Laminated limestone \\
\hline $34-1,15-16$ & 352.65 & 82 & Laminated limestone \\
\hline $34-1,69-70$ & 293.69 & 90 & Nannofossil chalk (olive-gray) \\
\hline $35-1,37-38$ & 362.37 & 84 & Laminated limestone \\
\hline $35-1,42-43$ & 302.92 & 85 & Nannofossil chalk (olive-gray) \\
\hline $36-2,120-121$ & 374.20 & 85 & Laminated limestone \\
\hline $38-2,15-16$ & 392.15 & 84 & Laminated limestone \\
\hline $39-2,34-35$ & 401.85 & 83 & Laminated limestone \\
\hline $40-1,108-109$ & 410.58 & 81 & Massive gray limestone \\
\hline
\end{tabular}

of $\mathrm{CaCO}_{3}$ in the gray limestones intercalated with the typical olive-gray laminated limestone of Unit II tend to be lower, because of increased silicification of these limestones.

Most of the samples of olive laminated limestone from Lithologic Unit II have high concentrations of organic carbon relative to concentrations commonly observed in pelagic carbonate sediments and rocks, with a maximum observed value of $8.6 \%$ (Fig. 4). The section containing unusually high concentrations of organic carbon in Hole 465A is 136 meters thick and spans about $5 \mathrm{~m} . \mathrm{y}$., from late Albian to early Cenomanian.

\section{Site 466}

The concentrations of $\mathrm{CaCO}_{3}$ in nannofossil oozes from Lithologic Unit IA are slightly lower than those in nannofossil oozes from Lithologic Unit IB, although concentrations are relatively high in both units (gener- 

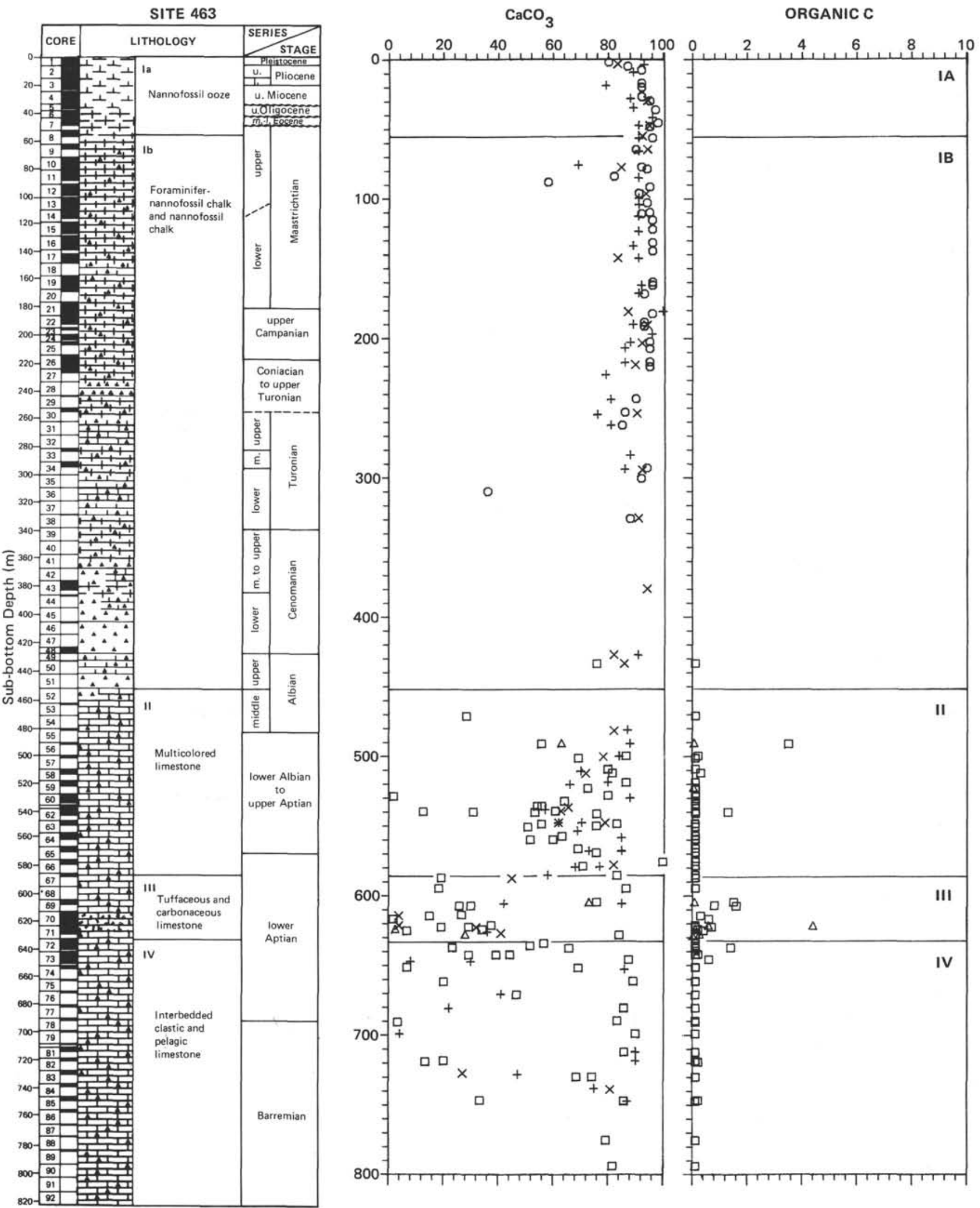

Figure 1. Concentrations of $\mathrm{CaCO}_{3}$ and organic carbon in samples from Hole 463. Organic-carbon concentrations were determined with a Leco car-

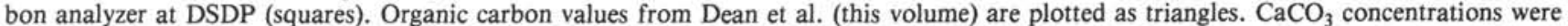
determined by Leco at DSDP (squares), by Leco at the University of Oslo (circles), or by shipboard carbonate-bomb (plus signs). Additional values of $\mathrm{CaCO}_{3}$ from Dean et al. (this volume) are plotted as triangles, and from Muller et al. (this volume) as X-symbols. 


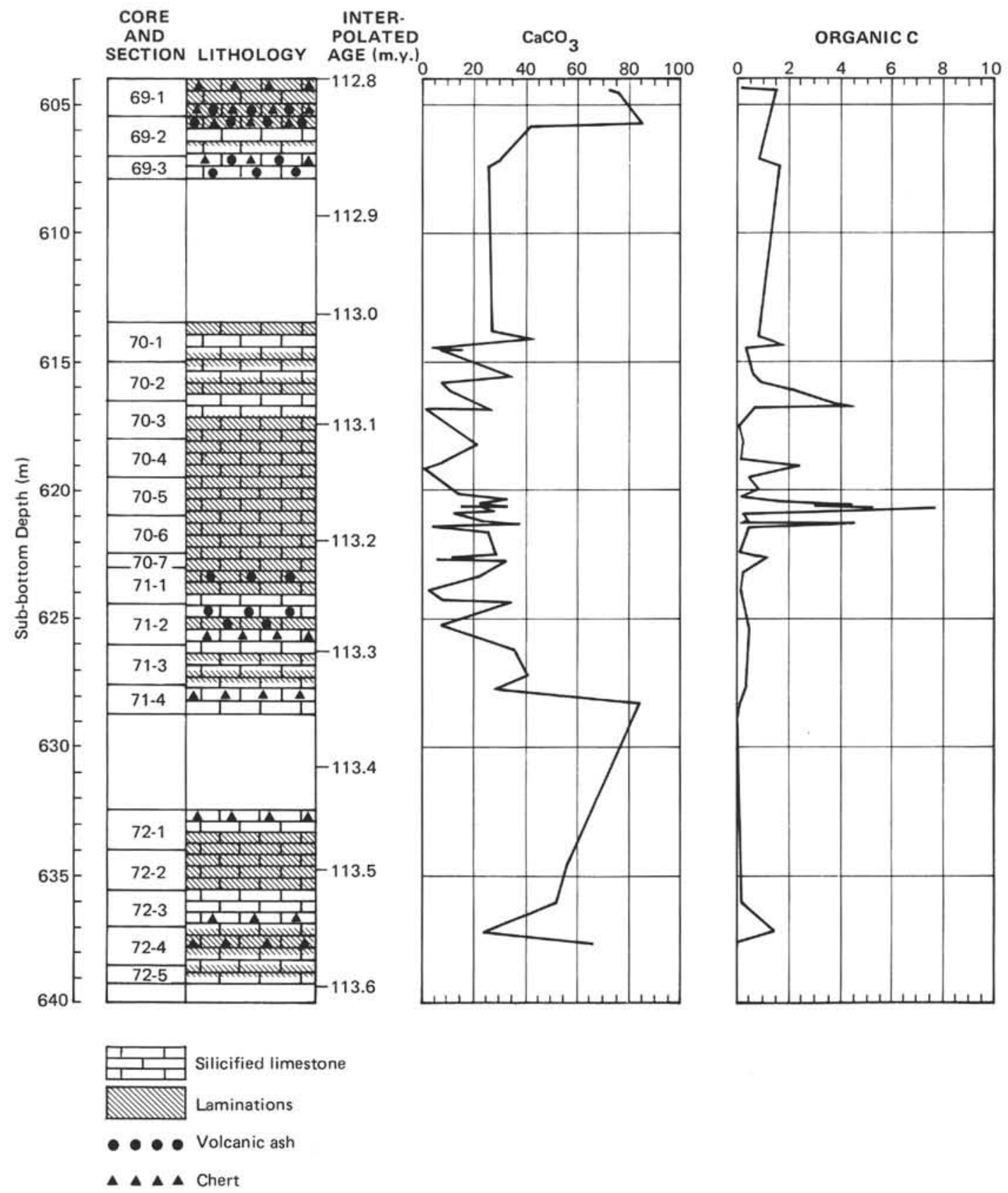

Figure 2. Lithologic summary and concentrations of $\mathrm{CaCO}_{3}$ and organic carbon in Cores 69 through 72, Hole 463.

ally greater than $88 \%$; Fig. 5). The lower concentrations of $\mathrm{CaCO}_{3}$ in Lithologic Unit IA, particularly in the upper part of the unit, are probably due to dilution by siliceous microfossils. Concentrations of $\mathrm{CaCO}_{3}$ in samples of olive-gray limestone from Lithologic Unit II range between 6 and $95 \%$, which is similar to the range of values observed for concentrations of $\mathrm{CaCO}_{3}$ in olive-gray limestones of equivalent age at Site 465 .

The nannofossil chalk and limestone of Lithologic Unit II contains up to $7.5 \%$ organic carbon and is the lateral equivalent of the organic-carbon-rich laminated limestone at Site 465 .

\section{REFERENCES}

Boyce, R. E., and Bode, G. W., 1972. Carbon and carbonate analyses, Leg 9 Deep Sea Drilling Project, In Hays, J. D., et al., Init. Repts. $D S D P$, 9: Washington (U.S. Govt. Printing Office), 797-816.

Müller, G., and Gastner, M., 1971. The "Karbonate Bombe"; A simple device for the determination of the carbonate content in sediments, soils and other materials: N. Jahrb. Mineral., Monatschefte, 10:466-469. 

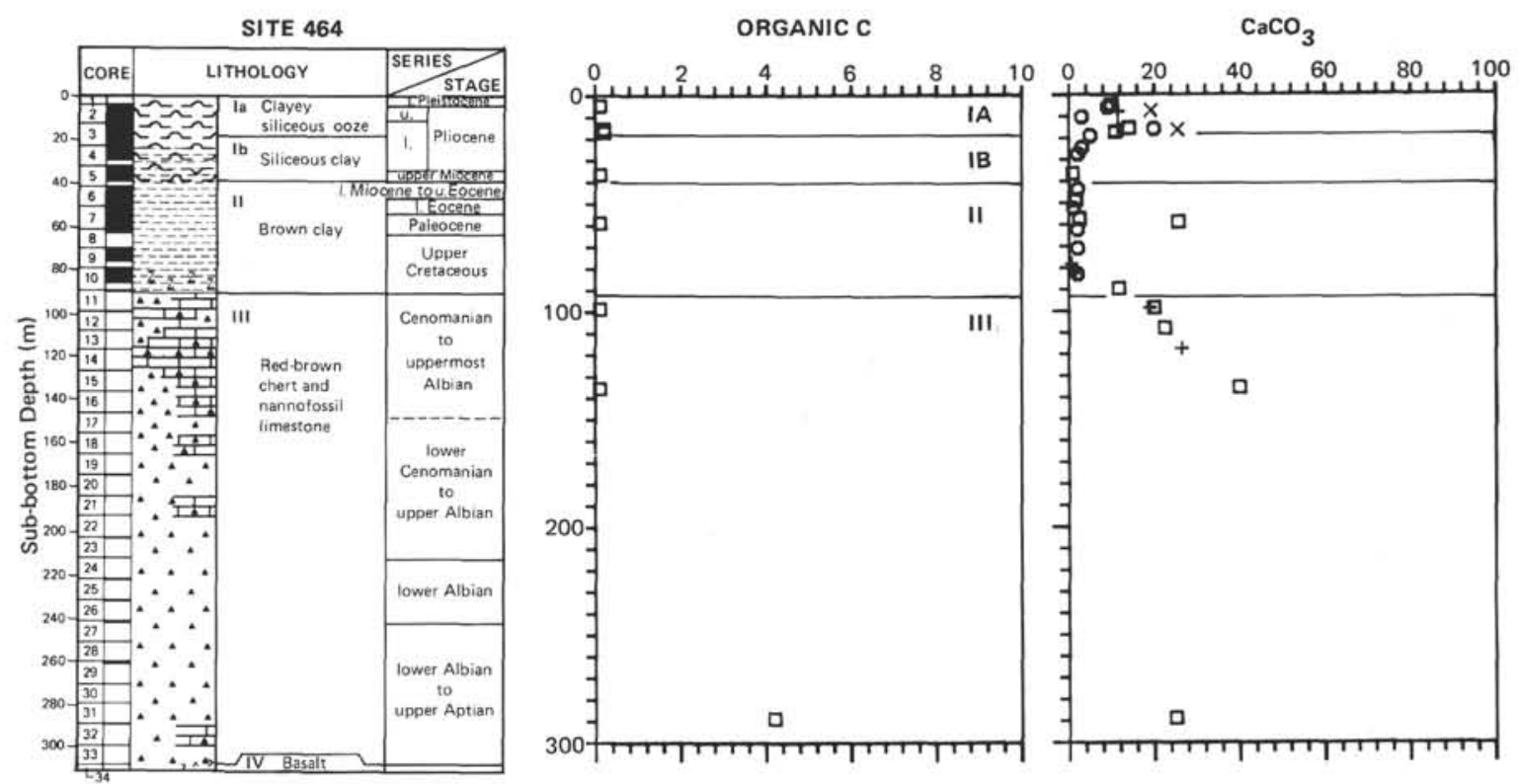

Figure 3. Concentrations of $\mathrm{CaCO}_{3}$ and organic carbon in samples from Hole 464. Organic-carbon concentrations were determined with a Leco carbon analyzer at DSDP (squares). Concentrations of $\mathrm{CaCO}_{3}$ were determined by Leco at DSDP (squares), carbonate-bomb (plus signs). Additional values of $\mathrm{CaCO}_{3}$ from Müller et al. (this volume) are plotted as $\mathrm{X}$-symbols.

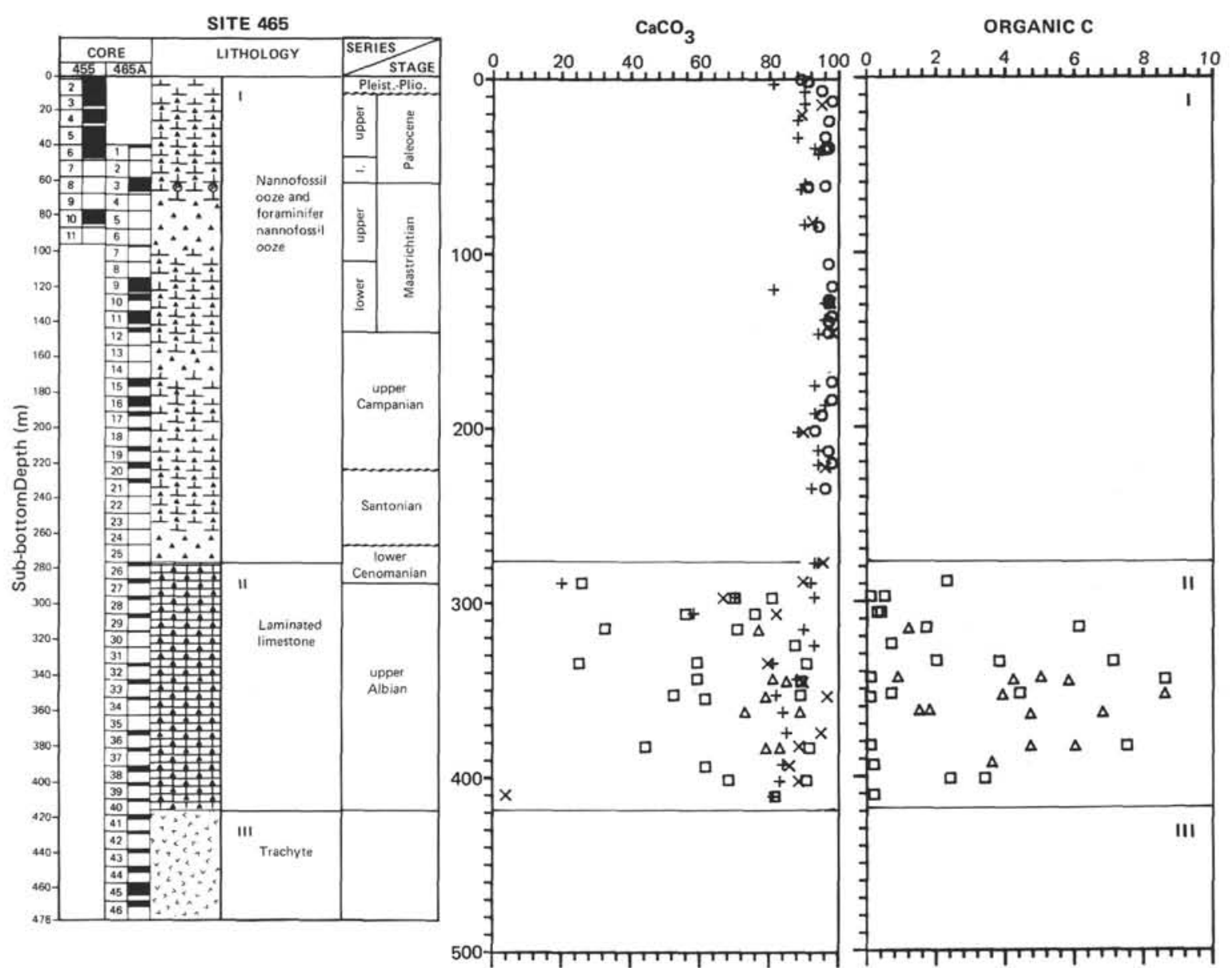

Figure 4. Concentrations of $\mathrm{CaCO}_{3}$ and organic carbon in samples from Holes 465 and 465A. Organic carbon concentrations were determined with a Leco carbon analyzer at DSDP (squares). Organic-carbon values from Dean et al. (this volume) are plotted as triangles. $\mathrm{CaCO}_{3}$ concentrations were determined by Leco at DSDP (squares), by Leco at the University of Oslo (circles), or by shipboard carbonate bomb (plus signs). Additional values of $\mathrm{CaCO}_{3}$ and organic carbon from Dean et al. (this volume) are plotted as triangles, and from Müller et al. (this volume) as X-symbols. 

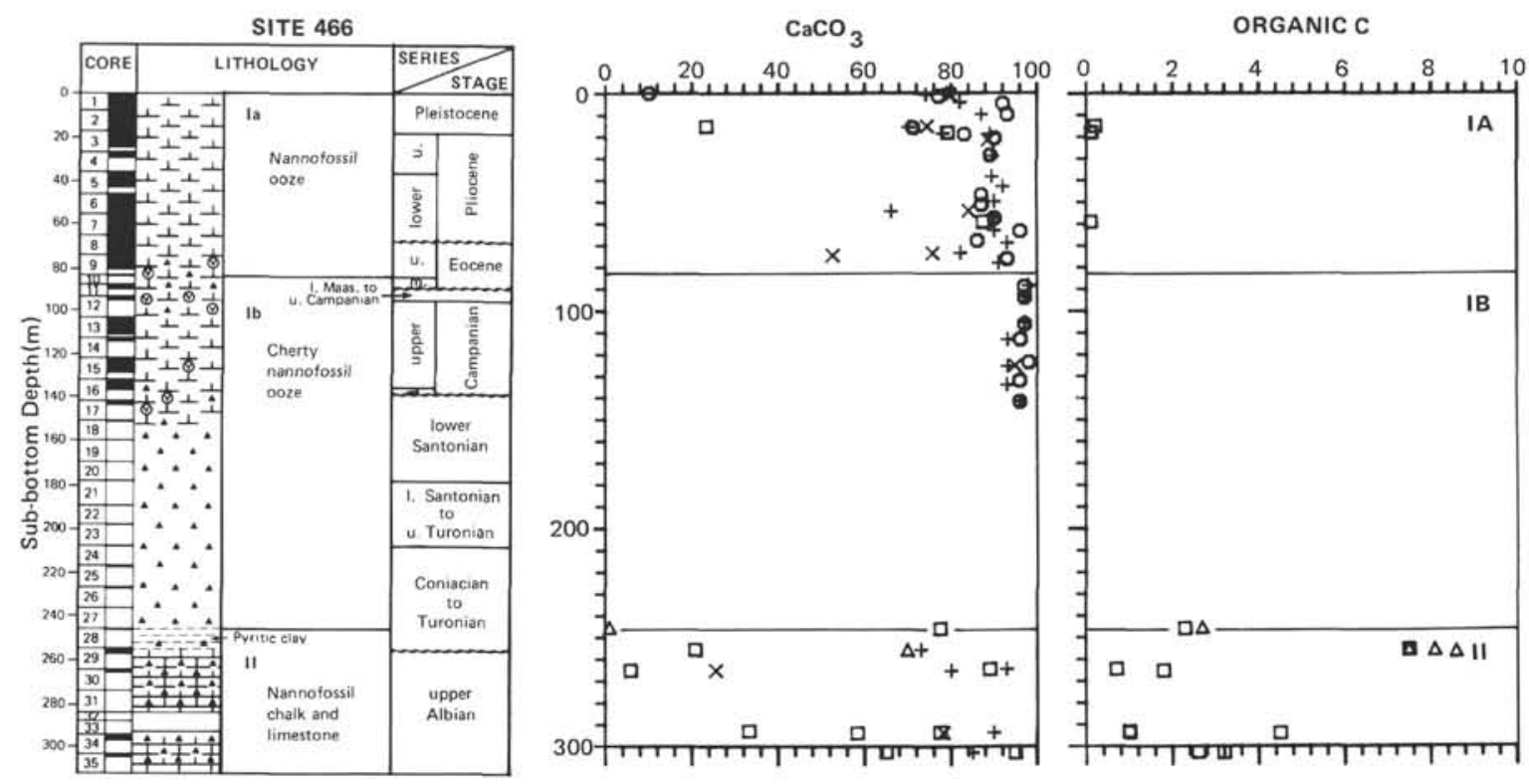

Figure 5. Concentrations of $\mathrm{CaCO}_{3}$ and organic carbon in samples from Hole 466. Organic-carbon concentrations were determined with a Leco carbon analyzer at DSDP (squares). Organic-carbon values from Dean et al. (this volume) are plotted as triangles. $\mathrm{CaCO}_{3}$ concentrations were determined by Leco at DSDP (squares), by Leco at the University of Oslo (circles), or by shipboard carbonate bomb (plus signs). Additional values of $\mathrm{CaCO}_{3}$ and organic carbon from Dean et al. (this volume) are plotted as triangles, and from Müller et al. (this volume) as X-symbols.

Table 2. Results of analyses for $\mathrm{CaCO}_{3}$ and organic carbon in samples from Sites $463,464,465$, and 466 , by Leco carbon analyzer at Deep Sea Drilling Project.

\begin{tabular}{|c|c|c|c|}
\hline Sample & $\begin{array}{l}\text { Sub-bottom } \\
\text { Depth } \\
\text { (m) }\end{array}$ & $\underset{(\%)}{\mathrm{CaCO}_{3}}$ & $\begin{array}{c}\text { Organic } C \\
(\%)\end{array}$ \\
\hline $463-50-1,39 \mathrm{~cm}$ & 433.39 & 75.8 & 0.1 \\
\hline $53, \mathrm{CC}, 26$ & 471.00 & 28.3 & 0.1 \\
\hline $56-1,95$ & 490.95 & 55.8 & 3.5 \\
\hline $57-1,16$ & 499.66 & 86.6 & 0.2 \\
\hline $57-2,20$ & 501.20 & 69.1 & 0.1 \\
\hline $58-1,10$ & 509.10 & 80.0 & 0.1 \\
\hline $58-3,10$ & 512.00 & 81.6 & 0.3 \\
\hline $59-1,18$ & 518.68 & 86.6 & 0.1 \\
\hline $59-3,139$ & 522.89 & 72.5 & 0.1 \\
\hline $60-1,18$ & 528.00 & 80.0 & 0.1 \\
\hline $60-1,78$ & 528.78 & 1.7 & 0.1 \\
\hline $60-3,110$ & 532.10 & 64.1 & 0.1 \\
\hline $61-1,20$ & 535.70 & 55.8 & 0.1 \\
\hline $61-1,28$ & 535.78 & 54.1 & 0.0 \\
\hline $62-2,14$ & 539.14 & 60.8 & 0.0 \\
\hline $62-2,71$ & 539.71 & 12.5 & 0.1 \\
\hline $62-2,90$ & 539.90 & 30.8 & 0.1 \\
\hline $62-2,116$ & 540.16 & 53.3 & 1.3 \\
\hline $62-3,64$ & 541.14 & 75.8 & 0.1 \\
\hline $63-1,128$ & 548.28 & 83.3 & 0.1 \\
\hline $63-2,2$ & 548.52 & 55.8 & 0.1 \\
\hline $63-2,128$ & 549.78 & 75.8 & 0.0 \\
\hline $62, \mathrm{CC}, 10$ & 550.65 & 50.8 & 0.1 \\
\hline $64-1,68$ & 557.18 & 63.3 & 0.1 \\
\hline $64-3,9$ & 559.59 & 60.0 & 0.1 \\
\hline $64-3,29$ & 559.79 & 51.7 & 0.1 \\
\hline $65-1,14$ & 566.14 & 69.1 & 0.1 \\
\hline $65-3,7$ & 569.07 & 75.8 & 0.1 \\
\hline $66-1,10$ & 575.60 & 100.0 & 0.1 \\
\hline $66-3,10$ & 578.60 & 70.8 & 0.1 \\
\hline $67-1,25$ & 585.25 & 83.3 & 0.1 \\
\hline $67-2,74$ & 587.24 & 19.2 & 0.1 \\
\hline
\end{tabular}

Table 2. (Continued).

\begin{tabular}{|c|c|c|c|}
\hline Sample & $\begin{array}{l}\text { Sub-bottom } \\
\text { Depth } \\
\text { (m) }\end{array}$ & $\begin{array}{c}\mathrm{CaCO}_{3} \\
(\%)\end{array}$ & $\underset{(\%)}{\text { Organic } C}$ \\
\hline $68-1,16$ & 594.66 & 86.6 & 0.1 \\
\hline $68-1,22$ & 594.72 & 18.3 & 0.1 \\
\hline $69-1,46$ & 604.46 & 75.8 & 1.5 \\
\hline $69-3,16$ & 607.16 & 30.0 & 0.8 \\
\hline $69-3,41$ & 607.41 & 25.8 & 1.6 \\
\hline $70-1,32$ & 613.82 & 26.7 & 0.0 \\
\hline $70-1,100$ & 614.50 & 15.0 & 0.3 \\
\hline $70-3,33$ & 616.83 & 1.7 & 0.6 \\
\hline $70-6,31$ & 621.31 & 37.5 & 0.1 \\
\hline $70, \mathrm{CC}, 0$ & 622.50 & 29.2 & 0.1 \\
\hline $70, \mathrm{CC}, 16$ & 622.66 & 19.2 & 0.7 \\
\hline $71-1,133$ & 624.33 & 34.2 & 0.2 \\
\hline $71-2,74$ & 625.24 & 6.7 & 0.4 \\
\hline $71-4,78$ & 628.28 & 84.1 & 0.1 \\
\hline $72-2,37$ & 634.37 & 56.6 & 0.1 \\
\hline $72-3,60$ & 636.10 & 51.6 & 0.1 \\
\hline $72-4,24$ & 637.24 & 23.3 & 1.4 \\
\hline $72-4,65$ & 637.65 & 65.8 & 0.1 \\
\hline $73-1,36$ & 642.36 & 44.2 & 0.2 \\
\hline $73-1,49$ & 642.49 & 39.2 & 0.1 \\
\hline $73-1,59$ & 642.56 & 29.2 & 0.0 \\
\hline $73-3,98$ & 645.98 & 87.5 & 0.6 \\
\hline $74-1,10$ & 651.50 & 6.7 & 0.1 \\
\hline $74-1,30$ & 651.80 & 69.1 & 0.1 \\
\hline $75-1,51$ & 661.51 & 89.1 & 0.1 \\
\hline $75-1,80$ & 661.80 & 20.0 & 0.1 \\
\hline $76-1,70$ & 671.20 & 46.7 & 0.1 \\
\hline $77-1,60$ & 680.60 & 85.8 & 0.1 \\
\hline $78-1,34$ & 689.84 & 83.3 & 0.1 \\
\hline $78-1,118$ & 690.68 & 3.3 & 0.1 \\
\hline $79-1,7$ & 699.07 & 90.0 & 0.1 \\
\hline $81-2,18$ & 712.18 & 85.8 & 0.1 \\
\hline $82-1,48$ & 718.48 & 20.0 & 0.1 \\
\hline $82-1,115$ & 719.15 & 13.3 & 0.2 \\
\hline $83-2,89$ & 729.89 & 74.1 & 0.1 \\
\hline
\end{tabular}


Table 2. (Continued).

\begin{tabular}{|c|c|c|c|}
\hline Sample & $\begin{array}{l}\text { Sub-bottom } \\
\text { Depth } \\
\text { (m) }\end{array}$ & $\begin{array}{c}\mathrm{CaCO}_{3} \\
(\%)\end{array}$ & $\begin{array}{c}\text { Organic C } \\
(\%)\end{array}$ \\
\hline $83-2,93$ & 729.93 & 68.3 & 0.1 \\
\hline $85-1,18$ & 746.68 & 33.3 & 0.2 \\
\hline $85-1,79$ & 747.29 & 85.8 & 0.1 \\
\hline $88-1,34$ & 775.34 & 79.1 & 0.1 \\
\hline $90-1,1$ & 794.01 & 81.6 & 0.1 \\
\hline $464-2-1,128$ & 4.78 & 10.0 & 0.1 \\
\hline $3-2,99$ & 15.49 & 14.2 & 0.2 \\
\hline $3-3,99$ & 16.99 & 10.9 & 0.2 \\
\hline $5-4,10$ & 36.60 & 0.8 & 0.1 \\
\hline $6-5,130$ & 48.80 & 1.7 & 0.0 \\
\hline $7-5,42$ & 57.49 & 2.5 & 0.0 \\
\hline $7-6,42$ & 58.92 & 25.8 & 0.1 \\
\hline $11-1,68$ & 89.68 & 11.7 & 0.0 \\
\hline $12-1,20$ & 98.70 & 20.0 & 0.1 \\
\hline $13, \mathrm{CC}, 10$ & 108.10 & 22.5 & 0.0 \\
\hline $16, \mathrm{CC}, 8$ & 135.66 & 40.0 & 0.1 \\
\hline $32-1,25$ & 288.75 & 25.0 & 4.2 \\
\hline $465-27, C C, 16$ & 288.50 & 25.8 & 2.3 \\
\hline $28-2,9$ & 297.09 & 80.8 & 0.5 \\
\hline $28-2,15$ & 297.15 & 70.0 & 0.1 \\
\hline $29-1,110$ & 306.10 & 75.8 & 0.3 \\
\hline $29-1,118$ & 306.18 & 55.8 & 0.4 \\
\hline $30-1,23$ & 314.73 & 32.5 & 6.1 \\
\hline $30-1,58$ & 315.08 & 70.8 & 1.7 \\
\hline $31-1,14$ & 324.14 & 87.5 & 0.7 \\
\hline $32-1,38$ & 333.88 & 59.1 & 2.0 \\
\hline $32-1,84$ & 334.34 & 25.0 & 7.1 \\
\hline $32-1,96$ & 334.46 & 90.8 & 3.8 \\
\hline $33-1,30$ & 343.30 & 59.1 & 0.1 \\
\hline $33-2,20$ & 344.70 & 89.1 & 8.6 \\
\hline $34-1,9$ & 352.59 & 89.1 & 0.7 \\
\hline $35-10,10$ & 352.60 & 52.5 & 4.4 \\
\hline $36-2,64$ & 354.64 & 61.6 & 0.1 \\
\hline $37-1,112$ & 382.12 & 44.2 & 0.1 \\
\hline $37-2,21$ & 382.71 & 91.6 & 7.5 \\
\hline $38-2,136$ & 393.36 & 61.6 & 0.2 \\
\hline $39-1,111$ & 401.11 & 68.3 & 2.4 \\
\hline $39-1,121$ & 401.21 & 90.8 & 3.4 \\
\hline $40-1,111$ & 410.61 & 81.6 & 0.2 \\
\hline $466-2-5,117$ & 15.17 & 23.3 & 0.2 \\
\hline $3-1,58$ & 18.08 & 79.1 & 0.1 \\
\hline $7-3,58$ & 59.08 & 87.5 & 0.1 \\
\hline $28, C C, 20$ & 246.00 & 77.5 & 2.3 \\
\hline $29-1,72$ & 255.72 & 20.8 & 7.5 \\
\hline $30-1,12$ & 264.62 & 89.1 & 0.7 \\
\hline $30-1,81$ & 265.31 & 5.8 & 1.8 \\
\hline $34-1,4$ & 293.04 & 33.3 & 1.0 \\
\hline $34-1,48$ & 293.48 & 77.5 & 1.0 \\
\hline $34-1,99$ & 293.99 & 58.3 & 4.5 \\
\hline $35-1,25$ & 302.75 & 65.0 & 2.6 \\
\hline $35-1,52$ & 303.02 & 95.0 & 3.2 \\
\hline
\end{tabular}

Table 3. Results of analyses for $\mathrm{CaCO}_{3}$ in samples from Sites 463, 464, 465, and 466 , by Leco carbon analyzer at the University of Oslo.

\begin{tabular}{ccc}
\hline Sample & $\begin{array}{c}\text { Sub-bottom } \\
\text { Depth } \\
(\mathrm{m})\end{array}$ & $\begin{array}{c}\mathrm{CaCO}_{3} \\
(\%)\end{array}$ \\
\hline $463-1-2,44 \mathrm{~cm}$ & 1.94 & 80 \\
$1-4,44$ & 4.94 & 87 \\
$2-2,54$ & 7.54 & 92 \\
\hline
\end{tabular}

Table 3. (Continued).

\begin{tabular}{|c|c|c|}
\hline Sample & $\begin{array}{l}\text { Sub-bottom } \\
\text { Depth } \\
\text { (m) }\end{array}$ & $\begin{array}{c}\mathrm{CaCO}_{3} \\
(\%)\end{array}$ \\
\hline $3-2,42$ & 16.92 & 92 \\
\hline $2-4,42$ & 19.92 & 92 \\
\hline $4-2,66$ & 26.66 & 92 \\
\hline $4-4,65$ & 29.65 & 95 \\
\hline $5-2,50$ & 36.00 & 97 \\
\hline $7-2,52$ & 45.52 & 98 \\
\hline $7-4,20$ & 48.20 & 95 \\
\hline $8-3,21$ & 56.21 & 96 \\
\hline $9-2,72$ & 64.72 & 90 \\
\hline $10-4,52$ & 77.02 & 92 \\
\hline $10-5,42$ & 78.42 & 94 \\
\hline $11-2,82$ & 83.82 & 82 \\
\hline $11-5,58$ & 88.08 & 58 \\
\hline $12-1,24$ & 91.24 & 95 \\
\hline $12-4,40$ & 95.90 & 91 \\
\hline $13-2,69$ & 102.69 & 94 \\
\hline $13-7,8$ & 109.60 & 95 \\
\hline $14-1,46$ & 110.46 & 92 \\
\hline $14-4,46$ & 114.96 & 96 \\
\hline $15-2,59$ & 121.59 & 96 \\
\hline $16-2,62$ & 131.12 & 96 \\
\hline $16-6,61$ & 137.11 & 96 \\
\hline $19-2,50$ & 159.50 & 96 \\
\hline $19-4,70$ & 161.70 & 96 \\
\hline $20-1,85$ & 167.85 & 93 \\
\hline $21-4,110$ & 182.10 & 96 \\
\hline $22-2,58$ & 188.08 & 93 \\
\hline $22-4,56$ & 191.06 & 93 \\
\hline $24-2,121$ & 202.21 & 95 \\
\hline $25-2,51$ & 207.01 & 95 \\
\hline $26-2,57$ & 216.57 & 95 \\
\hline $26-4,102$ & 220.02 & 95 \\
\hline $29-1,12$ & 243.12 & 90 \\
\hline $30-1,30$ & 252.80 & 86 \\
\hline $31-1,4$ & 262.04 & 85 \\
\hline $34-2,68$ & 292.68 & 94 \\
\hline $35-1,15$ & 300.15 & 92 \\
\hline $36-1,10$ & 309.60 & 36 \\
\hline $38-1,62$ & 329.12 & 88 \\
\hline $464-2-2,75$ & 5.75 & 9 \\
\hline $2-5,91$ & 10.41 & 3 \\
\hline $3-2,144$ & 15.92 & 20 \\
\hline $3-4,144$ & 18.94 & 5 \\
\hline $4-2,65$ & 24.65 & 3 \\
\hline $4-4,75$ & 27.75 & 2 \\
\hline $5-2,100$ & 34.50 & 0 \\
\hline $5-4,100$ & 37.50 & 0 \\
\hline $6-2,88$ & 43.88 & 2 \\
\hline $7-2,15$ & 52.65 & 1 \\
\hline $8-2,45$ & 62.45 & 2 \\
\hline $9-1,115$ & 71.15 & 2 \\
\hline $10-3,60$ & 83.10 & 2 \\
\hline $465-1-1,30$ & 0.30 & 89 \\
\hline $2-1,57$ & 1.57 & 91 \\
\hline $2-4,57$ & 6.57 & 95 \\
\hline $3-2,65$ & 12.65 & 98 \\
\hline $4-3,103$ & 24.03 & 97 \\
\hline $5-3,70$ & 33.20 & 96 \\
\hline $6-1,105$ & 40.05 & 96 \\
\hline $10-5,106$ & 84.06 & 94 \\
\hline $1-1,34$ & 39.34 & 97 \\
\hline $3-3,14$ & 61.14 & 96 \\
\hline $3-3,90$ & 61.90 & 91 \\
\hline $8-1,14$ & 105.64 & 97 \\
\hline $9-3,51$ & 118.51 & 98 \\
\hline $10-2,29$ & 126.29 & 97 \\
\hline $10-3,29$ & 127.79 & 97 \\
\hline
\end{tabular}


Table 3. (Continued).

\begin{tabular}{|c|c|c|}
\hline Sample & $\begin{array}{l}\text { Sub-bottom } \\
\text { Depth } \\
\text { (m) }\end{array}$ & $\begin{array}{c}\mathrm{CaCO}_{3} \\
(\%)\end{array}$ \\
\hline $11-2,10$ & 135.60 & 98 \\
\hline $11-4,10$ & 138.60 & 97 \\
\hline $12-2,10$ & 145.10 & 97 \\
\hline $15-1,106$ & 173.06 & 98 \\
\hline $16-2,33$ & 183.33 & 98 \\
\hline $17-1,114$ & 192.14 & 95 \\
\hline $18-1,55$ & 201.05 & 93 \\
\hline $19-2,131$ & 212.81 & 97 \\
\hline $20-1,18$ & 219.68 & 98 \\
\hline $21-4,71$ & 234.21 & 96 \\
\hline $466-1-1,134$ & 1.34 & 77 \\
\hline $1-4,23$ & 4.73 & 92 \\
\hline $2-2,41$ & 9.91 & 93 \\
\hline $2-6,37$ & 15.87 & 71 \\
\hline $3-1,136$ & 18.86 & 83 \\
\hline $3-3,20$ & 20.70 & 90 \\
\hline $4-2,14$ & 28.64 & 89 \\
\hline $5-1,127$ & - & 10 \\
\hline $6-1,77$ & 46.77 & 87 \\
\hline $6-4,77$ & 51.27 & 87 \\
\hline $7-2,44$ & 57.44 & 90 \\
\hline $7-6,44$ & 63.44 & 96 \\
\hline $8-2,135$ & 67.85 & 86 \\
\hline $9-2,30$ & 76.30 & 93 \\
\hline $11-1,97$ & 88.97 & 97 \\
\hline $12-1,52$ & 94.02 & 97 \\
\hline $13-3,20$ & 106.20 & 97 \\
\hline $14-1,69$ & 113.19 & 96 \\
\hline $15-2,20$ & 123.70 & 98 \\
\hline $16-1,67$ & 132.17 & 96 \\
\hline $17-1,89$ & 141.89 & 96 \\
\hline
\end{tabular}

Table 4. Results of analyses for $\mathrm{CaCO}_{3}$ and organic carbon in samples from Site 463 by Leco carbon analyzer at the University of Oslo.

\begin{tabular}{|c|c|c|c|}
\hline Sample & $\begin{array}{l}\text { Sub-bottom } \\
\text { Depth } \\
\text { (m) }\end{array}$ & $\begin{array}{c}\mathrm{CaCO}_{3} \\
(\%)\end{array}$ & $\begin{array}{c}\text { Organic } \\
\text { Carbon } \\
(\%)\end{array}$ \\
\hline $463-70-1,60 \mathrm{~cm}$ & 614.10 & 42.4 & 0.80 \\
\hline $70-1,92$ & 614.42 & 10.8 & 1.70 \\
\hline $70-1,102$ & 614.52 & 7.4 & 0.30 \\
\hline $70-2,58$ & 615.58 & 34.9 & 0.60 \\
\hline $70-2,84$ & 615.84 & 7.4 & 0.80 \\
\hline $70-2,116$ & 616.16 & 11.6 & 2.10 \\
\hline $70-3,21$ & 616.71 & 22.4 & 3.70 \\
\hline $70-3,32$ & 616.82 & 25.8 & 4.40 \\
\hline $70-3,102$ & 617.52 & 12.0 & 0.05 \\
\hline $70-4,17$ & 618.17 & 20.8 & 0.20 \\
\hline $70-4,85$ & 618.85 & 8.3 & 0.10 \\
\hline $70-4,112$ & 619.12 & 0.8 & 2.30 \\
\hline $70-5,5$ & 619.55 & 6.6 & 0.40 \\
\hline $70-5,48$ & 619.98 & 13.3 & 0.80 \\
\hline $70-5,60$ & 620.10 & 14.9 & 0.50 \\
\hline $70-5,80$ & 620.30 & 32.4 & 0.20 \\
\hline $70-5,101$ & 620.51 & 23.3 & 2.00 \\
\hline $70-5,110$ & 620.60 & 32.4 & 4.40 \\
\hline $70-5,115$ & 620.65 & 15.8 & 3.00 \\
\hline $70-5,120$ & 620.70 & 25.8 & 5.10 \\
\hline $70-5,125$ & 620.75 & 26.6 & 4.60 \\
\hline $70-5,130$ & 620.80 & 27.4 & 7.50 \\
\hline $70-5,135$ & 620.85 & 24.9 & 4.80 \\
\hline $70-5,140$ & 620.90 & 12.4 & 1.80 \\
\hline $70-5,145$ & 620.95 & 13.3 & 0.20 \\
\hline $70-6,16$ & 621.16 & 22.4 & 0.40 \\
\hline $70-6,41$ & 621.41 & 9.1 & 1.70 \\
\hline $70-6,47$ & 621.47 & 11.6 & 0.40 \\
\hline $70-6,60$ & 621.60 & 25.8 & 0.40 \\
\hline $70-7,14$ & 622.64 & 10.8 & 0.00 \\
\hline $70, \mathrm{CC}, 20$ & 622.70 & 6.6 & 1.20 \\
\hline $71-1,30$ & 623.30 & 22.4 & 0.20 \\
\hline $71-1,97$ & 623.97 & 3.3 & 0.00 \\
\hline $71-1,124$ & 624.24 & 7.4 & 0.00 \\
\hline
\end{tabular}

